\title{
On the spectral gap of some Cayley graphs on the Weyl group $W\left(B_{n}\right)$
}

\author{
Filippo Cesi \\ Dipartimento di Fisica, Università di Roma "La Sapienza", Italy.
}

\begin{abstract}
The Laplacian of a (weighted) Cayley graph on the Weyl group $W\left(B_{n}\right)$ is a $N \times N$ matrix with $N=2^{n} n$ ! equal to the order of the group. We show that for a class of (weighted) generating sets, its spectral gap (lowest nontrivial eigenvalue), is actually equal to the spectral gap of a $2 n \times 2 n$ matrix associated to a $2 n$-dimensional permutation representation of $W_{n}$. This result can be viewed as an extension to $W\left(B_{n}\right)$ of an analogous result valid for the symmetric group, known as "Aldous' spectral gap conjecture", proven in 2010 by Caputo, Liggett and Richthammer.
\end{abstract}

Keywords: Cayley graph, spectral gap, Aldous' conjecture, Weyl group, Coxeter group, Laplacian matrix

2000 MSC: 05C25, 05C50, 20C15, 20C30, 60K35

Email address: filippo.cesi@roma1.infn.it (Filippo Cesi) 


\title{
On the spectral gap of some Cayley graphs on the Weyl group $W\left(B_{n}\right)$
}

\author{
Filippo Cesi
}

Dipartimento di Fisica, Università di Roma "La Sapienza", Italy.

\section{Introduction}

Let $G$ be a finite group with complex group algebra $\mathbb{C} G$. If $w=\sum_{g \in G} w_{g} g$ is an element of the group algebra such that all coefficients $w_{g}$ are real, nonnegative and symmetric, i.e. $w_{g^{-1}}=w_{g}$, we denote by $\operatorname{Cay}(G, w)$ the weighted Cayley graph whose vertices are the elements of $G$ and whose (undirected) edges are the pairs $\{g, h g\}$ with $g, h \in G$. Each edge $\{g, h g\}$ carries a weight equal to $w_{h}$. The Laplacian of $\operatorname{Cay}(G, w)$ is a linear operator acting on functions $f: G \rightarrow \mathbb{C}$ as

$$
\left[\Delta_{\text {Cay }(G, w)} f\right](g)=\sum_{h \in G} w_{h}(f(g)-f(h g)) .
$$

Since (weighted) Cayley graphs are regular, the Laplacian is strictly related to the (weighted) adjacency matrix $A_{\mathrm{Cay}(G, w)}$, namely

$$
\Delta_{\mathrm{Cay}(G, w)}=\left(\sum_{g \in G} w_{g}\right) I_{N}-A_{\mathrm{Cay}(G, w)},
$$

where $N$ is the order of $G$ and $I_{N}$ is the $N \times N$ identity matrix. The lowest eigenvalue of the Laplacian is trivially zero with a constant eigenvector. The spectral gap of $\operatorname{Cay}(G, w)$ is defined as the second lowest eigenvalue of the associated Laplacian and it is denoted by $\psi_{G}(w)$. It is strictly positive if and only if the support of $w$ generates $G$, that is if $\operatorname{Cay}(G, w)$ is connected.

Email address: filippo.cesi@roma1.infn.it (Filippo Cesi) 
Taking into account the symmetry of $w$, we can rewrite the Laplacian as

$$
\Delta_{\text {Cay }(G, w)}=\sum_{h \in G} w_{h}\left[I_{N}-\mathbf{L}(h)\right]
$$

where $\mathbf{L}$ is the left regular representation of $G$ acting on functions on $G$ as

$$
[\mathbf{L}(h) f](g)=f\left(h^{-1} g\right) .
$$

Formula (1.1) suggests the following generalization: Given a representation $\mathbf{R}$ of $G$ on the $d$-dimensional complex vector space $V$, and given $w \in \mathbb{C} G$, following [1], we define the representation Laplacian $\Delta_{G}(w, \mathbf{R})$ as the linear operator on $V$ given by

$$
\Delta_{G}(w, \mathbf{R}):=\sum_{h \in G} w_{h}\left[I_{V}-\mathbf{R}(h)\right] \quad w_{h} \in \mathbb{C},
$$

where $I_{V}$ is the identity on $V$. To the pair $(w, \mathbf{R})$ we also associate a spectral gap, denoted by $\psi_{G}(w, \mathbf{R})$, which is again the smallest nontrivial eigenvalue of $\Delta_{G}(w, \mathbf{R})$ (see Section 2 for a precise definition). Thus the Laplacian of the Cayley graph is a special case of the representation Laplacian, and we can write

$$
\Delta_{\text {Cay }(G, w)}=\Delta_{G}(w, \mathbf{L}) \quad \psi_{G}(w)=\psi_{G}(w, \mathbf{L}) .
$$

In this paper we pursue the general idea of [1] that, although $\mathbf{L}$ contains all irreducible representations of $G$, in some interesting cases it is possible to pinpoint those representations which are "responsible" for the spectral gap of the Cayley graph. These representations can then replace $\mathbf{L}$ in (1.4), with the advantage of having to deal with a possibly much smaller matrix than $\Delta_{\text {Cay }(G, w)}$.

The most important result so far in this direction is the proof of the so called Aldous' spectral gap conjecture, concerning the symmetric group. After several partial successes in a series of papers [2], [3], [4], [5], [6], [7], [8], [9], [10] spanning about 25 years, a general proof was finally given in [11]. While the original formulation was given in a probabilistic framework [12], the statement can be translated as follows (see [9] for more details on the equivalence): let $\mathfrak{S}_{n}$ be the symmetric group on $\{1, \ldots, n\}$, and let $T_{n}$ be the set of all transpositions in $\mathfrak{S}_{n}$.

Theorem 1.1. (Aldous' spectral gap conjecture, proven in [11]). Let $w \in$ $\mathbb{C S}_{n}$ be given by

$$
w=\sum_{(i j) \in T_{n}} b_{i j}(i j)
$$


where $(i j)$ is the transposition which exchanges $i$ and $j$, and $b_{i j} \geq 0$. Then

$$
\psi_{G}(w)=\psi_{G}\left(w, \mathbf{D}_{n}^{0}\right)
$$

where $\mathbf{D}_{n}^{0}$ is the $n$-dimensional defining representation of $\mathfrak{S}_{n}$, associated with the natural action of $\mathfrak{S}_{n}$ on the set $\{1,2, \ldots, n\}$.

Thanks to this theorem, if $w$ is supported on transpositions, in order to find the spectral gap of the Laplacian of $\operatorname{Cay}\left(\mathfrak{S}_{n}, w\right)$, which is a $n ! \times n !$ matrix, all one has to do is to find the smallest nontrivial eigenvalue of a $n \times n$ matrix.

The spectral gap of Cayley graphs on the symmetric or alternating groups has been computed also in some particular cases where the generators are not transpositions. Examples are the initial reversal graph [13], the (complete, extended) alternating group graph [14] and the substring reversal graph [15]. In [16] the authors prove that if $w=\sum_{\pi \in[\alpha]} \pi$, where $[\alpha]$ is a conjugacy class of $\mathfrak{S}_{n}$, then, for $n$ large enough, the spectral gap of $w$ is associated with one of eight low-dimensional representations. A weaker statement is also proven for the more general case where $w$ is invariant under conjugation.

In [1] we described a general strategy for proving results analogous to Theorem 1.1 for arbitrary finite groups and we gave a slightly simpler proof based on this point of view. In particular, in this strategy, it appears that the representations "responsible" for the spectral gap are the nontrivial irreducible representations of $\mathfrak{S}_{n}$ which, when restricted to $\mathfrak{S}_{n-1}$, contain the trivial representation. In the case of the symmetric group there is a unique such representation, namely the one associated with the partition $(n-1,1)$. This representation, apart from a trivial summand, is equivalent to $\mathbf{D}_{n}^{0}$ (see $(3.6))$.

In this paper we apply the idea of [1] to the Weyl/Coxeter group $W\left(B_{n}\right)$ associated with the $B_{n}$ (or $C_{n}$ ) root system, also called the hyperoctahedral group. For simplicity we let $W_{n}=W\left(B_{n}\right)$. There are several equivalent ways to define this group. One possible realizationis as the subgroup of $G L(n, \mathbb{C})$ consisting of all $n \times n$ matrices which have exactly one non-zero entry in each row and each column, and this non-zero entry is either 1 or -1 . We have thus a natural embedding $\mathfrak{S}_{n} \hookrightarrow W_{n}$ where the symmetric group is the subgroup of all matrices with nonnegative entries. The group $W_{n}$ can also be described as a group of signed permutations. This leads to another embedding

$$
\mathbf{P}_{n}: W_{n} \hookrightarrow \mathfrak{S}_{2 n},
$$


where $\mathbf{P}_{n}$ is a $2 n$-dimensional faithful permutation representation described in more details in Section 3.

We can now state the main result of this paper: for $i \in\{1, \ldots, n\}$, let $s_{\{i\}} \in W_{n}$ be the diagonal matrix ${ }^{1}$

$$
s_{\{i\}}=\operatorname{diag}(1, \ldots, 1,-1,1, \ldots, 1),
$$

where the unique -1 occurs in the $i^{\text {th }}$ place. Then we have:

Theorem 1.2. Let $w \in \mathbb{C} W_{n}$ be given by

$$
w=\sum_{i=1}^{n} a_{i} s_{\{i\}}+\sum_{(i j) \in T_{n}} b_{i j}(i j) \quad a_{i} \geq 0, b_{i j} \geq 0 .
$$

Then

$$
\psi_{W_{n}}(w)=\psi_{W_{n}}\left(w, \mathbf{P}_{n}\right)
$$

In our approach the representation $\mathbf{P}_{n}$ appears in (1.8) for the same reason that the defining representation of the symmetric group appears in (1.5), that is $\mathbf{P}_{n}$ contains all irreducible representations of $W_{n}$ which, when restricted to $W_{n-1}$, contain the trivial representation.

There is another result which is worth mentioning since it has an interesting overlap with Theorem 1.2. In [17] it is proved that if $(G, S)$ is a finite Coxeter system, then both the spectral gap and the Kazhdan constant are determined by the defining representation. In our notation, this implies that if $G=W_{n}$ and if $w$ is the sum of a Coxeter generating set, that is

$$
w=s_{\{1\}}+\sum_{i=1}^{n-1}(i, i+1)
$$

then $\psi_{W_{n}}(w)=\psi_{W_{n}}\left(w, \mathbf{D}_{n}\right)$, where $\mathbf{D}_{n}$ is the $n$-dimensional defining representation of $W_{n}$ which will be defined in Section 3. This result covers every finite Coxeter group, but applies (essentially) to only one element of the group algebra, namely $w=\sum_{s \in S} s$, where $S$ is a Coxeter generator for $G$. The approach used in the proof of this theorem is very different from ours,

\footnotetext{
${ }^{1}$ In this introduction we are implicitly using the defining representation for describing the elements of $W_{n}$. See (4.8). This particular realization of $W_{n}$ will be denoted with $\widetilde{W}_{n}$ in Section 3.
} 
and in Section 5 of [1] we explain why it is unlikely that it could be effective for dealing with more general elements $w$ of the group algebra. Since $\mathbf{D}_{n}$ is a subrepresentation of $\mathbf{P}_{n}$ as we show in Proposition 3.1, Kassabov's result is stronger than ours for $w$ of the form (1.9). Nevertheless, (1.8) is optimal for a general $w$ of type (1.7). In Section 5 we show, in fact, that the theorem is (in general) false if we try to improve it by replacing $\mathbf{P}_{n}$ with a subrepresentation. We also discuss a possible generalization of Theorem 1.2 .

\section{The representation Laplacian and its spectral gap}

If $G$ is a finite group, $\operatorname{rep}(G)$ denotes the set of all finite-dimensional complex representations of $G$, while $\operatorname{Irr}(G)$ is the set of all equivalence classes of irreducible representations. By Maschke's theorem, we have for each $\mathbf{R} \in$ $\operatorname{rep}(G)$,

$$
\mathbf{R} \cong \bigoplus_{\mathbf{T} \in \operatorname{Irr}(G)} \mu(\mathbf{R}, \mathbf{T}) \mathbf{T}
$$

where $\mu(\mathbf{R}, \mathbf{T})$ is a nonnegative integer called the multiplicity of $\mathbf{T}$ in $\mathbf{R}$. If $\mathbf{R}$ is a representation of $G$ on the complex vector space $V, V^{G, \mathbf{R}}$ stands for the subspace of all invariant vectors

$$
V^{G, \mathbf{R}}=\{v \in V: \mathbf{R}(g) v=v, \forall g \in G\} .
$$

By definition we have

$$
\operatorname{dim} V^{G, \mathbf{R}}=\mu(\mathbf{R}, \mathbf{I}),
$$

where $\mathbf{I}$ is the one-dimensional trivial representation. An eigenvalue $\lambda$ of the representation Laplacian $\Delta_{G}(w, \mathbf{R})$, defined in (1.3), will be called trivial if its corresponding eigenspace consists entirely of invariant vectors $v \in V^{G, \mathbf{R}}$.

If $w \in \mathbb{C} G$, the support of $w$ is defined as

$$
\operatorname{supp} w=\left\{g \in G: w_{g} \neq 0\right\} .
$$

We introduce a canonical involution in the group algebra $\mathbb{C} G$ as

$$
w=\sum_{g \in G} w_{g} g \longrightarrow w^{*}:=\sum_{g \in G} \bar{w}_{g} g^{-1} .
$$


An element $w \in \mathbb{C} G$ is called symmetric if $w=w^{*}$, and it is called positive if $w_{g} \geq 0$ for all $g \in G$. We let

$$
\begin{aligned}
\mathbb{C} G^{(s)} & =\{w \in \mathbb{C} G: w \text { is symmetric }\} \\
\mathbb{R}_{+} G^{(s)} & =\{w \in \mathbb{C} G: w \text { is symmetric and positive }\} .
\end{aligned}
$$

It is easy to prove $\left[1\right.$, Sect. 2] that $\Delta_{G}(w, \mathbf{R})$ has real eigenvalues if $w$ is symmetric and real nonnegative eigenvalues if $w$ is symmetric and positive. If $w$ is symmetric we can label the eigenvalues of $\Delta_{G}(w, \mathbf{R})$, with possible repetitions according to their multiplicity, in nondecreasing order as

$$
\lambda_{1}\left(\Delta_{G}(w, \mathbf{R})\right) \leq \lambda_{2}\left(\Delta_{G}(w, \mathbf{R})\right) \leq \cdots \leq \lambda_{s}\left(\Delta_{G}(w, \mathbf{R})\right),
$$

where $s$ is the degree (or dimension) of the representation $\mathbf{R}$. If $w \in \mathbb{R}_{+} G^{(s)}$, we define the spectral gap of the pair $(w, \mathbf{R})$ as

$$
\psi_{G}(w, \mathbf{R}):=\min \left\{\lambda \in \operatorname{spec} \Delta_{G}(w, \mathbf{R}): \lambda \text { is nontrivial }\right\}
$$

with the convention that $\min \emptyset=+\infty$. If $t=\operatorname{dim} V^{G, \mathbf{R}}$, then $\Delta_{G}(w, \mathbf{R})$ has exactly $t$ trivial eigenvalues, thus, thanks to $(2.2)$, we have ${ }^{2}$

$$
\psi_{G}(w, \mathbf{R})=\lambda_{t+1}\left(\Delta_{G}(w, \mathbf{R})\right) \quad \text { where } t=\mu(\mathbf{R}, \mathbf{I}) .
$$

The spectral gap of $w$ is defined by minimizing over representations

$$
\psi_{G}(w)=\inf \left\{\psi_{G}(w, \mathbf{R}): \mathbf{R} \in \operatorname{rep}(G)\right\} .
$$

From (2.1) it follows that

$$
\operatorname{spec} \Delta_{G}(w, \mathbf{R})=\bigcup_{\mathbf{T} \in \operatorname{Irr}(G): \mu(\mathbf{R}, \mathbf{T})>0} \operatorname{spec} \Delta_{G}(w, \mathbf{T})
$$

which implies

$$
\psi_{G}(w, \mathbf{R})=\min \left\{\psi_{G}(w, \mathbf{T}): \mathbf{T} \in \operatorname{Irr}(G), \mu(\mathbf{R}, \mathbf{T})>0\right\} .
$$

Consequently in (2.5) we can just consider irreducible representations, so

$$
\psi_{G}(w)=\min \left\{\psi_{G}(w, \mathbf{R}): \mathbf{R} \in \operatorname{Irr}(G)\right\} .
$$

\footnotetext{
${ }^{2}$ Unless $\mathbf{R}$ is a multiple of $\mathbf{I}$ in which case all eigenvalues are trivial, and thus the spectral gap is equal to $+\infty$.
} 
Let $\mathbf{L}$ be the left regular representation of $G$ defined in (1.2). Since

$$
\mathbf{L}=\bigoplus_{\mathbf{T} \in \operatorname{Irr}(G)} f_{\mathbf{T}} \mathbf{T}
$$

where $f_{\mathbf{T}}$ is the degree of $\mathbf{T}$, we have $\mu(\mathbf{L}, \mathbf{I})=1$. Therefore $\psi_{G}(w)=$ $\psi_{G}(w, \mathbf{L})=\lambda_{2}\left(\Delta_{G}(w, \mathbf{L})\right)$. This shows that definitions (2.5) and (2.8) for the quantity $\psi_{G}(w)$ actually agree with the definition given in Section 1 as the second lowest eigenvalue of the Cayley graph $\operatorname{Cay}(G, w)$.

\section{Groups $\mathfrak{S}_{n}, W\left(B_{n}\right)$ and their representations}

In this section we review some more or less well known facts about the symmetric group, the Weyl (or Coxeter) group $W_{n}:=W\left(B_{n}\right)$ and their representations. For more details we refer the reader to [18], [19], [20].

A partition of $n$ is a nonincreasing sequence $\alpha=\left(\alpha_{1}, \alpha_{2}, \ldots, \alpha_{r}\right)$ of positive integers such that $\sum_{i=1}^{r} \alpha_{i}=n$. The size of $\alpha$ is defined as $|\alpha|=\sum_{i=1}^{r} \alpha_{i}$. We write $\alpha \vdash n$ if $\alpha$ is a partition of $n$. The irreducible representations of $\mathfrak{S}_{n}$ are indexed (modulo equivalence) by the partitions of $n$. If $\alpha \vdash n$, we denote by $[\alpha]$ the corresponding irreducible representation of $\mathfrak{S}_{n}$.

The group $W_{n}$ can be realized as the set of all pairs $(\eta, \pi)$ with $\eta \in\{0,1\}^{n}$ and $\pi \in \mathfrak{S}_{n}$ with product

$$
(\eta, \pi) \cdot(\zeta, \sigma)=\left(\eta+\zeta \circ \pi^{-1}, \pi \sigma\right)
$$

where the sum of two elements of $\{0,1\}^{n}$ is componentwise $\bmod 2$, and elements of $\{0,1\}^{n}$ are identified with functions from $\{1, \ldots, n\}$ to $\{0,1\}$. Observe that $(\eta, \pi)=\left(\eta, 1_{\mathfrak{S}_{n}}\right) \cdot(0, \pi)=(0, \pi) \cdot\left(\eta \circ \pi, 1_{\mathfrak{S}_{n}}\right)$. Consider the two subgroups

$$
\begin{aligned}
N_{n} & :=\left\{\left(\eta, 1_{\mathfrak{S}_{n}}\right): \eta \in\{0,1\}^{n}\right\} \cong(\mathbb{Z} / 2 \mathbb{Z})^{n} \\
S_{n} & :=\left\{(0, \pi): \pi \in \mathfrak{S}_{n}\right\} \cong \mathfrak{S}_{n}
\end{aligned}
$$

$N_{n}$ is a normal subgroup of $W_{n}$ and $W_{n}$ can be written as a semidirect product

$$
W_{n}=N_{n} \rtimes S_{n} \cong(\mathbb{Z} / 2 \mathbb{Z})^{n} \rtimes \mathfrak{S}_{n} .
$$

The irreducible representations of $W_{n}$ are indexed by ordered pairs of partitions $(\alpha, \beta)$ such that $|\alpha|+|\beta|=n$. We denote by $[\alpha, \beta]$ the irreducible representation corresponding to $(\alpha, \beta)$. We also denote by $\mathbf{T}^{\alpha}$ and $\mathbf{T}^{(\alpha, \beta)}$ 
some specific (but arbitrary) choice of representations in the equivalence classes $[\alpha]$ and $[\alpha, \beta]$ respectively. Given a pair of partitions $(\alpha, \beta)$ with $|\alpha|=k$ and $|\beta|=n-k$, the representation $[\alpha, \beta]$ can be obtained [20, Sect. 2] as an induced representation $\mathrm{as}^{3}$

$$
[\alpha, \beta] \cong\left(\mathbf{U}_{k} \otimes[\alpha] \otimes[\beta]\right) \uparrow_{N_{n} \cdot\left(S_{k} \times S_{n-k}\right)}^{W_{n}},
$$

where $\mathbf{U}_{k}$ is the one-dimensional representation of $N_{n}$ given by

$$
\mathbf{U}_{k}(\eta)=(-1)^{\operatorname{card}\left\{i \in\{k+1, \ldots, n\}: \eta_{i}=1\right\}} \quad \eta \in\{0,1\}^{n}, k \in\{0, \ldots, n\} .
$$

In particular, when $k=n$ (and thus $\beta=\emptyset$ ), we have that $\mathbf{U}_{n}$ is the trivial representation and $[\alpha, \emptyset]$ is the pullback of the $[\alpha]$ representation of $S_{n} \cong$ $W_{n} / N_{n}$, that is

$$
\mathbf{T}^{(\alpha, \emptyset)}(\eta, \pi)=\mathbf{T}^{\alpha}(\pi) \quad(\eta, \pi) \in W_{n} .
$$

The trivial representation of $W_{n}$ is given by $\mathbf{I}_{n}=[(n), \emptyset]$.

Branching rules. An irreducible representation of a finite group is in general no longer irreducible when restricted to a subgroup, but it can be expressed as a direct sum of irreducible representations of the subgroup. The branching rule $\mathfrak{S}_{n} \rightarrow \mathfrak{S}_{n-1}$ is $[18$, Sect. 6.1 .8$]$

$$
[\alpha] \downarrow_{\mathfrak{S}_{n-1}}^{\mathfrak{S}_{n}}=\bigoplus_{\beta \in \alpha^{-}}[\beta] \quad \alpha \vdash n
$$

where, if $\alpha=\left(\alpha_{1}, \ldots, \alpha_{r}\right), \alpha^{-}$is defined as the collection of all sequences of the form

$$
\left(\alpha_{1}, \ldots, \alpha_{i-1}, \alpha_{i}-1, \alpha_{i+1}, \ldots, \alpha_{r}\right)
$$

which are partitions of $n-1$. The branching rule $W_{n} \rightarrow W_{n-1}$ is $[18$, Sect. $6.1 .9]$

$$
[\alpha, \beta] \downarrow_{\mathcal{W}_{n-1}}^{\mathcal{W}_{n}}=\bigoplus_{\gamma \in \alpha^{-}}[\gamma, \beta] \oplus \bigoplus_{\gamma \in \beta^{-}}[\alpha, \gamma]
$$

\footnotetext{
${ }^{3}$ we use the same notation as [18], while in [20] the order of $[\alpha, \beta]$ is reversed. It is easy to verify that although the group product $N_{n} \cdot\left(S_{k} \times S_{n-k}\right)$ is not a direct product, nevertheless $\mathbf{U}_{k} \otimes[\alpha] \otimes[\beta]$ is indeed a representation. The interested reader is referred to [21, Sect. 8.2], where the construction of the irreducible representations of semidirect products by abelian groups is explained.
} 
The defining representation of $\mathfrak{S}_{n}$. Let $\mathbf{D}_{n}^{0}$ be the defining $n$-dimensional representation of $\mathfrak{S}_{n}$ with matrix elements

$$
\left[\mathbf{D}_{n}^{0}(\pi)\right]_{i j}=\delta_{i, \pi(j)} \quad \pi \in \mathfrak{S}_{n} .
$$

This representation is not irreducible, but it can be decomposed as

$$
\mathbf{D}_{n}^{0}=(n) \oplus(n-1,1)
$$

The defining representation of $W_{n}$. We let $\mathbf{D}_{n}$ be the $n$-dimensional defining representation of $W_{n}$ given by

$$
\left[\mathbf{D}_{n}(\eta, \pi)\right]_{i j}=(-1)^{\eta_{i}} \delta_{i, \pi(j)}
$$

This is a faithful representation, hence $W_{n}$ is isomorphic to the image of $\mathbf{D}_{n}$ which is the group $\widetilde{W}_{n}$ of all $n \times n$ matrices which have exactly one non-zero entry in each row and each column, and this non-zero entry is either 1 or -1 . The normal subgroup $N_{n}$ is mapped to the subgroup of the diagonal matrices of $\widetilde{W}_{n}$, while the restriction of $\mathbf{D}_{n}$ to $S_{n}$ is just the $n$-dimensional defining representation of $\mathfrak{S}_{n}$. As explained right before Proposition II.1 of [20] (see also [18, Proposition 5.5.7] for a more general statement) $\mathbf{D}_{n}$ is irreducible, in particular,

$$
\mathbf{D}_{n} \cong[(n-1),(1)]
$$

The representation $\widetilde{\mathbf{D}}_{n}^{0}$. Since $N_{n}$ is normal in $W_{n}$, every representation $\mathbf{R}$ of the quotient $W_{n} / N_{n} \cong \mathfrak{S}_{n}$ can be pulled back (or lifted) to a representation $\widetilde{\mathbf{R}}$ of $W_{n}$ letting

$$
\widetilde{\mathbf{R}}(\eta, \pi)=\mathbf{R}(\pi) \quad(\eta, \pi) \in W_{n} .
$$

Furthermore $\widetilde{\mathbf{R}}$ is irreducible if and only if $\mathbf{R}$ is. We define $\widetilde{\mathbf{D}}_{n}^{0}$ as the pullback of the defining $n$-dimensional representation of $\mathfrak{S}_{n}$. Its matrix elements are then

$$
\left[\widetilde{\mathbf{D}}_{n}^{0}(\eta, \pi)\right]_{i j}=\delta_{i, \pi(j)} .
$$

From (3.6) and (3.2) it follows that

$$
\widetilde{\mathbf{D}}_{n}^{0}=[(n), \emptyset] \oplus[(n-1,1), \emptyset] .
$$


The permutation representation $\mathbf{P}_{n}$. Let $X_{n}=\{-n, \ldots,-1\} \cup\{1, \ldots, n\}$ and consider the (left) group action of $W_{n}$ on $X_{n}$ given by

$$
(\eta, \pi) k=(-1)^{(\eta \circ \pi)(|k|)} \operatorname{sgn}(k) \pi(|k|) \quad k \in X_{n} .
$$

We define $\mathbf{P}_{n}$ as the $2 n$-dimensional permutation representation associated with this action. $\mathbf{P}_{n}$ acts on the complex vector space

$$
V_{n}=\mathbb{C} X_{n}:=\left\{\left(x_{i}\right)_{i \in X_{n}}: x_{i} \in \mathbb{C}\right\} .
$$

If $\left(e_{i}\right)_{i \in X_{n}}$ is the canonical basis of $V_{n}$, such that

$$
\sum_{i \in X_{n}} x_{i} e_{i}=\left(x_{-n}, \ldots, x_{-1}, x_{1}, \ldots, x_{n}\right),
$$

the representation matrices are determined by the equalities

$$
\mathbf{P}_{n}(g) e_{k}=e_{g k} \quad g \in W_{n}, k \in X_{n},
$$

where $g k$ is given by (3.12). Therefore the matrix elements of $\mathbf{P}_{n}$ are given by

$$
\left[\mathbf{P}_{n}(\eta, \pi)\right]_{i j}= \begin{cases}1 & \text { if } \pi(|j|)=|i| \text { and } \operatorname{sgn}(j)=\operatorname{sgn}(i)(-1)^{\eta_{|i|}} \\ 0 & \text { otherwise }\end{cases}
$$

This representation is also faithful, so $W_{n}$ is isomorphic to the image of $\mathbf{P}_{n}$ which consists of the set of all permutations $\pi$ of $X_{n}$ such that $\pi(-k)=$ $-\pi(k)$ for each $k \in X_{n}$ (the so called signed permutations).

In the following proposition we find the irreducible components of $\mathbf{P}_{n}$.

Proposition 3.1. We have

$$
\mathbf{P}_{n}=\mathbf{D}_{n} \oplus \widetilde{\mathbf{D}}_{n}^{0}=[(n-1),(1)] \oplus \mathbf{I}_{n} \oplus[(n-1,1), \emptyset] .
$$

Proof. Let $V_{n}=\mathbb{C} X_{n}$ and $\left(e_{i}\right)_{i \in X_{n}}$ be as above, and let

$$
e_{k}^{+}=e_{k}+e_{-k} \quad e_{k}^{-}=e_{k}-e_{-k} \quad k=1, \ldots, n .
$$

Let $V_{n}^{+}\left(V_{n}^{-}\right)$be the subspace of $V_{n}$ spanned by $\left(e_{k}^{+}\right)_{k=1}^{n}\left(\left(e_{k}^{-}\right)_{k=1}^{n}\right)$. In other words $V_{n}^{+}$is the subspace of the "even" vectors such that $x_{-i}=x_{i}$, while $V_{n}^{-}$is the subspace of the odd vectors.

Let $g=(\eta, \pi) \in W_{n}$. The action defined in (3.12) satisfies $g(-k)=$ $-g(k)$. Thus we have, for $k=1, \ldots, n$,

$$
\mathbf{P}_{n}(g) e_{k}^{+}=e_{g k}+e_{g(-k)}=e_{g k}+e_{-g k}=e_{|g k|}^{+}=e_{\pi(k)}^{+}
$$


and

$$
\begin{aligned}
\mathbf{P}_{n}(g) e_{k}^{-} & =e_{g k}-e_{g(-k)}=e_{g k}-e_{-g k}=\operatorname{sgn}(g k) e_{|g k|}^{-} \\
& =(-1)^{(\eta \circ \pi)(k)} e_{\pi(k)}^{-} .
\end{aligned}
$$

It follows that both $V_{n}^{+}$and $V_{n}^{-}$are invariant under $\mathbf{P}_{n}(g)$, thus we have a direct sum decomposition

$$
\mathbf{P}_{n}=\mathbf{P}_{n}^{+} \oplus \mathbf{P}_{n}^{-} \quad V_{n}=V_{n}^{+} \oplus V_{n}^{-} \quad \mathbf{P}_{n}^{ \pm}:=\left.\mathbf{P}_{n}\right|_{V_{n}^{ \pm}}
$$

By comparing (3.14), (3.15) with (3.10), (3.7), we obtain

$$
\mathbf{P}_{n}^{+}=\widetilde{\mathbf{D}}_{n}^{0} \quad \mathbf{P}_{n}^{-}=\mathbf{D}_{n} .
$$

The second equality in (3.13) follows from (3.8), (3.11).

\section{Proof of Theorem 1.2}

In this section we prove Theorem 1.2 following the strategy described in $[1$, Sect. 3].

Since $G$ is a finite group, we can always assume that the representations are unitary with respect to some (positive definite) inner product $\langle\cdot, \cdot\rangle$ defined on the representation space $V$. This will ensure that, if $w$ is a symmetric element of the group algebra, then $\Delta_{G}(w, \mathbf{R})$ is self-adjoint. For a self-adjoint linear operator $A$ we write $A \geq 0$ if $\langle A \cdot, \cdot\rangle$ is a positive semidefinite bilinear form. We will write $\Delta_{G}(w, \mathbf{R}) \geq 0$ if $\Delta_{G}(w, \mathbf{R})$ is positive semidefinite for some (equivalently for each) unitary version of $\mathbf{R}$. We can thus define

$$
\Gamma(G)=\left\{w \in \mathbb{C} G^{(s)}: \Delta_{G}(w, \mathbf{R}) \geq 0, \forall \mathbf{R} \in \operatorname{rep}(G)\right\} .
$$

For future reference we summarize a few elementary properties of the set $\Gamma(G)$ in the following proposition.

Proposition 4.1. We have:

(1) $\Gamma(G)$ is a convex cone, i.e. if $w, z \in \Gamma(G)$, then for any $\alpha, \beta \in \mathbb{R}_{+}$, $\alpha w+\beta z \in \Gamma(G)$

(2) $\Delta_{G}\left(w, \oplus_{i=1}^{n} \mathbf{R}_{i}\right) \geq 0$ if and only if $\Delta_{G}\left(w, \mathbf{R}_{i}\right) \geq 0$ for every $i=1, \ldots, n$;

(3) $\Gamma(G)=\left\{w \in \mathbb{C} G^{(s)}: \Delta_{G}(w, \mathbf{T}) \geq 0, \forall \mathbf{T} \in \operatorname{Irr}(G)\right\}$; 
(4) $\Gamma(G)=\left\{w \in \mathbb{C} G^{(s)}: \Delta_{G}(w, \mathbf{L}) \geq 0\right\}$, where $\mathbf{L}$ is the left regular representation of $G$;

(5) $\mathbb{R}_{+} G^{(s)} \subset \Gamma(G)$;

(6) If $H$ is a subgroup of $G$, then $\Gamma(H) \subset \Gamma(G)$.

Proof. (1) and (2) follow from the definitions. (3) follows from (2) and (2.1). (4) follows from (2), (3) and (2.9). If $\mathbf{R}$ is a unitary representation on $V$, and $w \in \mathbb{C} G^{(s)}$, a straightforward computation (see Proposition 2.1 in [1]) yields

$$
\left\langle\Delta_{G}(w, \mathbf{R}) v, v\right\rangle=\frac{1}{2} \sum_{g \in G} w_{g}\|\mathbf{R}(g) v-v\|^{2} \quad v \in V .
$$

Thus $\Delta_{G}(w, \mathbf{R}) \geq 0$ if $w$ is positive, which proves (5).

Finally, let $w \in \Gamma(H)$. Then $\Delta_{H}(w, \mathbf{T}) \geq 0$ for every $\mathbf{T} \in \operatorname{Irr}(H)$. If $\mathbf{S} \in \operatorname{Irr}(G)$, then we have a branching rule

$$
\mathbf{S} \downarrow_{H}^{G} \cong \bigoplus_{\mathbf{T} \in \operatorname{Irr}(H)} k(\mathbf{T}) \mathbf{T}
$$

where $k(\mathbf{T})$ are suitable nonnegative integers. Since $w$ is an element of the group algebra of $H$, the same decomposition applies to the representation Laplacian

$$
\Delta_{G}(w, \mathbf{S})=\bigoplus_{\mathbf{T} \in \operatorname{Irr}(H)} k(\mathbf{T}) \Delta_{H}(w, \mathbf{T})
$$

Therefore $\Delta_{G}(w, \mathbf{S}) \geq 0$ and (6) follows.

In the following we regard $W_{n-1}$ as the subgroup of $W_{n}$ which fixes the last coordinate, that is

$$
W_{n-1} \cong\left\{(\eta, \pi) \in W_{n}: \eta_{n}=0 \text { and } \pi(n)=n\right\} .
$$

The key point of the proof is the following "semirecursive" result:

Proposition 4.2. Let $w \in \mathbb{R}_{+} W_{n}^{(s)}$ and $z \in \mathbb{R}_{+} W_{n-1}^{(s)}$, be such that $w-z \in$ $\Gamma\left(W_{n}\right)$. Then

$$
\psi_{W_{n}}(w) \geq \min \left\{\psi_{W_{n-1}}(z), \psi_{W_{n}}\left(w, \mathbf{P}_{n}\right)\right\}
$$


Proof. Let $\mathcal{I}_{n}$ be the set of all irreducible representations of $W_{n}$ that, when restricted to $W_{n-1}$, contain the trivial representation. The branching rule (3.4) implies that

$$
\mathcal{I}_{n}=\left\{\mathbf{I}_{n},[(n-1,1), \emptyset],[(n-1),(1)]\right\} .
$$

Thanks to Proposition 3.1 and (2.7), and using the fact that $\psi_{G}(w, \mathbf{I})=+\infty$, we obtain

$$
\psi_{W_{n}}\left(w, \mathbf{P}_{n}\right)=\min \left\{\psi_{W_{n}}(w,[(n-1,1), \emptyset]), \psi_{W_{n}}(w,[(n-1),(1)])\right\} .
$$

Thus Proposition 4.2 follows from Proposition 3.2 in [1].

Let $\mathcal{A}_{n}$ be the subset of $\mathbb{R}_{+} W_{n}^{(s)}$ considered in the hypothesis of Theorem 1.2

$$
\mathcal{A}_{n}=\left\{\sum_{i=1}^{n} a_{i} s_{\{i\}}+\sum_{(i j) \in T_{n}} b_{i j}(i j): a_{i} \geq 0, b_{i j} \geq 0\right\} .
$$

If $w \in \mathcal{A}_{n}$, let us write $w=w_{N}+w_{T}$ with

$$
w_{N}=\sum_{i=1}^{n} a_{i} s_{\{i\}} \quad w_{T}=\sum_{(i j) \in T_{n}} b_{i j}(i j) .
$$

We observe that in the $(\eta, \pi)$ notation for the elements of $W_{n}$ we have

$$
s_{\{i\}}=\left(\eta_{\{i\}}, 1_{\mathfrak{S}_{n}}\right) \quad \text { where } \quad\left(\eta_{\{i\}}\right)_{j}=\delta_{i j}= \begin{cases}1 & \text { if } j=i \\ 0 & \text { if } j \neq i .\end{cases}
$$

It follows from (3.7) and (3.10) that

$$
\begin{aligned}
& \widetilde{\mathbf{D}}_{n}^{0}\left(s_{\{i\}}\right)=I_{n} \\
& \mathbf{D}_{n}\left(s_{\{i\}}\right)=\operatorname{diag}\left((-1)^{\delta_{i j}}\right)_{j=1}^{n} .
\end{aligned}
$$

Thus we get

$$
\begin{aligned}
& \Delta_{W_{n}}\left(w_{N}, \widetilde{\mathbf{D}}_{n}^{0}\right)=0 \\
& \Delta_{W_{n}}\left(w_{N}, \mathbf{D}_{n}\right)=2 \operatorname{diag}\left(a_{i}\right)_{i=1}^{n} \\
& \Delta_{W_{n}}\left(w_{T}, \widetilde{\mathbf{D}}_{n}^{0}\right)=\Delta_{W_{n}}\left(w_{T}, \mathbf{D}_{n}\right) .
\end{aligned}
$$

Strategy for proving Theorem 1.2.

Let us now assume that we find a map $\vartheta: \mathcal{A}_{n} \rightarrow \mathcal{A}_{n-1}$ such that the following holds for each $w \in \mathcal{A}_{n}$ : 
(a) $w-\vartheta(w) \in \Gamma\left(W_{n}\right)$;

(b) $\psi_{W_{n}}\left(w, \mathbf{P}_{n}\right) \leq \psi_{W_{n-1}}\left(\vartheta(w), \mathbf{P}_{n-1}\right)$.

Then we can prove (1.8) by induction. Assume in fact that (1.8) holds for $n=k-1$, that is

$$
\psi_{W_{k-1}}(z)=\psi_{W_{k-1}}\left(z, \mathbf{P}_{k-1}\right) \quad \forall z \in \mathcal{A}_{k-1} .
$$

From Proposition 4.2 and (4.12) with $z=\vartheta(w)$, and from properties (a), (b) of the map $\vartheta$ it follows that

$$
\psi_{W_{k}}(w) \geq \min \left\{\psi_{W_{k-1}}\left(\vartheta(w), \mathbf{P}_{k-1}\right), \psi_{W_{k}}\left(w, \mathbf{P}_{k}\right)\right\}=\psi_{W_{k}}\left(w, \mathbf{P}_{k}\right),
$$

which, combined with the reversed inequality which is a trivial consequence of (2.5), implies $\psi_{W_{k}}(w)=\psi_{W_{k}}\left(w, \mathbf{P}_{k}\right)$. The induction step is completed.

In the next proposition we take care of the starting point of the induction, $n=2$.

Proposition 4.3. If $w \in \mathcal{A}_{2}$, then $\psi_{W_{2}}(w)=\psi_{W_{2}}\left(w, \mathbf{P}_{2}\right)$.

Proof. We have

$$
\operatorname{Irr}\left(W_{2}\right)=\left\{\mathbf{I}_{2},[(1,1), \emptyset],[(1),(1)],[\emptyset,(2)],[\emptyset,(1,1)]\right\} .
$$

Proposition II.1 of $[20]$ states that if $[\alpha, \beta] \in \operatorname{Irr}\left(W_{n}\right)$, then

$$
[\beta, \alpha] \cong \mathbf{J}_{n} \widehat{\otimes}[\alpha, \beta]
$$

where $\widehat{\otimes}$ denotes the inner tensor product of representations and $\mathbf{J}_{n}=$ $[\emptyset,(n)]$ is the one-dimensional representation of $W_{n}$ given by

$$
\mathbf{J}_{n}(\eta, \pi)=(-1)^{\operatorname{card}\left\{i \in\{1, \ldots, n\}: \eta_{i}=1\right\}} \quad(\eta, \pi) \in W_{n} .
$$

Using (3.2) and (4.6), we have

$$
\begin{aligned}
& \mathbf{T}^{(\emptyset, \alpha)}\left(s_{\{i\}}\right)=\mathbf{J}_{n}\left(\eta_{\{i\}}, 1_{\mathfrak{S}_{n}}\right) \mathbf{T}^{\alpha}\left(1_{\mathfrak{S}_{n}}\right)=(-1) \mathbf{T}^{\alpha}\left(1_{\mathfrak{S}_{n}}\right)=-I_{d} \\
& \mathbf{T}^{(\emptyset, \alpha)}((i j))=\mathbf{J}_{n}(0,(i j)) \mathbf{T}^{\alpha}((i j))=\mathbf{T}^{\alpha}((i j)),
\end{aligned}
$$

where $d$ is the degree of $[\alpha]$. This implies that, for every $w \in \mathcal{A}_{n}$ of the form (4.4), we have

$$
\Delta_{W_{n}}(w,[\emptyset, \alpha])-\Delta_{W_{n}}(w,[\alpha, \emptyset])=\left(2 \sum_{i=1}^{n} a_{i}\right) I_{d}
$$


Therefore the eigenvalues of $\Delta_{W_{n}}(w,[\emptyset, \alpha])$ are shifted, with respect to the eigenvalues of $\Delta_{W_{n}}(w,[\alpha, \emptyset])$ by a nonnegative quantity. In particular, if $\alpha \neq(n)$, then $[\alpha, \emptyset]$ is nontrivial and it has a spectral gap which is not greater than the spectral gap of $[\emptyset, \alpha]$. For this reason, representations of type $[\emptyset, \alpha]$ with $\alpha \neq(n)$ can be safely omitted in the minimization process (2.8) which produces the spectral gap of $w$.

Going back to the case $n=2$, we can take care of the representation $[\emptyset,(2)]$ with an explicit calculation. If $w \in \mathcal{A}_{2}$, it can be written as

$$
w=x s_{\{1\}}+y s_{\{2\}}+z(12) \quad x, y, z \geq 0 .
$$

Since the Laplacian of the trivial representation is null, (4.16) becomes

$$
\Delta_{W_{2}}(w,[\emptyset,(2)])=2(x+y) I_{1} .
$$

On the other hand, using (3.7), we get

$$
\Delta_{W_{2}}\left(w, \mathbf{D}_{2}\right)=\left[\begin{array}{cc}
2 x+z & -z \\
-z & 2 y+z
\end{array}\right]=: B_{2}
$$

whose eigenvalues are

$$
x+y+z \pm\left((x-y)^{2}+z^{2}\right)^{1 / 2} .
$$

It follows that

$$
\begin{aligned}
\psi_{W_{2}}\left(w, \mathbf{D}_{2}\right) & =\lambda_{1}\left(B_{2}\right)=x+y+z-\left((x-y)^{2}+z^{2}\right)^{1 / 2} \\
& \leq x+y \leq 2(x+y)=\psi_{W_{2}}(w,[\emptyset,(2)]) .
\end{aligned}
$$

Thus, for the purpose of computing the spectral gap of $w$, representation $[\emptyset,(2)]$ can also be disregarded in the list (4.13). Consequently

$$
\psi_{W_{2}}(w)=\min \left\{\psi_{W_{2}}(w,[(1,1), \emptyset]), \psi_{W_{2}}(w,[(1),(1)])\right\}=\psi_{W_{2}}\left(w, \mathbf{P}_{2}\right) .
$$

The mapping $\vartheta$. In order to conclude the proof of Theorem 1.2 we are going to define a map $\vartheta: \mathcal{A}_{n} \rightarrow \mathcal{A}_{n-1}$ which satisfies properties (a) and (b) stated above.

If $w=\sum_{i=1}^{n} a_{i} s_{\{i\}}$ with $a_{i} \geq 0$, we let $\ell$ be the largest index $j$ such that $a_{j}=\min _{i} a_{i}$, and we define

$$
\vartheta^{N}(w)=\sum_{i=1, i \neq \ell}^{n} a_{i} s_{\{i\}} .
$$


If $w=\sum_{(i j) \in T_{n}} b_{i j}(i j)$ with $b_{i j} \geq 0$, for each $m=1, \ldots, n$ we let

$$
\vartheta_{m}^{T}(w)=\sum_{\substack{(i k) \in T_{n} \\ i, k \neq m}}\left[w_{i k}+\frac{w_{i m} w_{k m}}{\sum_{j \neq m} w_{j m}}\right](i k) .
$$

Finally we define a mapping $\vartheta(w)$ as follows: let $w=w_{N}+w_{T}$ as in (4.5). Then we let

$$
\vartheta(w)=\vartheta^{N}\left(w_{N}\right)+\vartheta_{\ell}^{T}\left(w_{T}\right)
$$

where $\ell$ is defined as above.

Remark 4.4. We can assume, without loss of generality, that $\ell=n$ in (4.19). In this way $\vartheta\left(\mathcal{A}_{n}\right) \subset \mathcal{A}_{n-1}$. Otherwise one can define $W_{n-1}$ as the subgroup of $W_{n}$ obtained by "dropping the $\ell^{t h}$ coordinate".

Remark 4.5. The mapping $\vartheta_{m}^{T}$, amazingly, appeared almost simultaneously in the preprint versions of [10] and [11] and it was key point which, together with a quite tricky inequality, called the "octopus inequality", produced a proof of Aldous' spectral gap conjecture in [11].

Properties (a) and (b) of the mapping $\vartheta$ will be proved in Lemmas 4.6 and 4.7 respectively, completing in this way the proof of Theorem 1.2.

Lemma 4.6. If $w \in \mathcal{A}_{n}$, then $w-\vartheta(w) \in \Gamma\left(W_{n}\right)$.

Proof. We can write

$$
w-\vartheta(w)=\underbrace{w_{N}-\vartheta^{N}\left(w_{N}\right)}_{\delta w_{N}}+\underbrace{w_{T}-\vartheta^{T}\left(w_{T}\right)}_{\delta w_{T}} .
$$

Since $\delta w_{N}$ is positive and symmetric, we have $\delta w_{N} \in \Gamma\left(W_{n}\right)$, thanks to Proposition 4.1(5).

On the other hand Theorem 2.3 of [11], the "octopus inequality" (see also Section 4 of [1] for a slightly simpler proof in which the algebraic perspective is more explicit), states that $\delta w_{T} \in \Gamma\left(\mathfrak{S}_{n}\right)$. By Proposition 4.1(6) we get $\delta w_{T} \in \Gamma\left(W_{n}\right)$.

Hence $w-\vartheta(w)=\delta w_{N}+\delta w_{T} \in \Gamma\left(W_{n}\right)$ by Proposition 4.1(1)

Lemma 4.7. If $w \in \mathcal{A}_{n}$, then

$$
\psi_{W_{n}}\left(w, \mathbf{P}_{n}\right) \leq \psi_{W_{n-1}}\left(\vartheta w, \mathbf{P}_{n-1}\right)
$$


Proof. From Proposition 3.1 we know that $\mathbf{P}_{n}=\widetilde{\mathbf{D}}_{n}^{0} \oplus \mathbf{D}_{n}$. Since $\mathbf{D}_{n}$ is irreducible, while $\widetilde{\mathbf{D}}_{n}^{0}$ contains the trivial representation with multiplicity 1 , by (2.4), we obtain

$$
\begin{aligned}
\psi_{W_{n}}\left(w, \mathbf{P}_{n}\right) & =\min \left\{\psi_{W_{n}}\left(w, \widetilde{\mathbf{D}}_{n}^{0}\right), \psi_{W_{n}}\left(w, \mathbf{D}_{n}\right)\right\} \\
& =\min \left\{\lambda_{2}\left(\Delta_{W_{n}}\left(w, \widetilde{\mathbf{D}}_{n}^{0}\right)\right), \lambda_{1}\left(\Delta_{W_{n}}\left(w, \mathbf{D}_{n}\right)\right)\right\}
\end{aligned}
$$

Since $\operatorname{supp}(\vartheta w) \in W_{n-1}$, the last row and column of its Laplacian are zero, thus we can write its representation Laplacian in block diagonal form as

$$
\begin{aligned}
& \Delta_{W_{n}}\left(\vartheta w, \widetilde{\mathbf{D}}_{n}^{0}\right)=\Delta_{W_{n-1}}\left(\vartheta w, \widetilde{\mathbf{D}}_{n-1}^{0}\right) \oplus[0]_{1 \times 1} \\
& \Delta_{W_{n}}\left(\vartheta w, \mathbf{D}_{n}\right)=\Delta_{W_{n-1}}\left(\vartheta w, \mathbf{D}_{n-1}\right) \oplus[0]_{1 \times 1},
\end{aligned}
$$

where $[x]_{1 \times 1}$ is the $1 \times 1$ matrix whose unique entry is equal to $x$. This implies

$$
\begin{aligned}
& \psi_{W_{n-1}}\left(\vartheta w, \mathbf{P}_{n-1}\right) \\
& \quad=\min \left\{\lambda_{2}\left(\Delta_{W_{n-1}}\left(\vartheta w, \widetilde{\mathbf{D}}_{n-1}^{0}\right)\right), \lambda_{1}\left(\Delta_{W_{n-1}}\left(\vartheta w, \mathbf{D}_{n-1}\right)\right)\right\} \\
& \quad=\min \left\{\lambda_{3}\left(\Delta_{W_{n}}\left(\vartheta w, \widetilde{\mathbf{D}}_{n}^{0}\right)\right), \lambda_{2}\left(\Delta_{W_{n}}\left(\vartheta w, \mathbf{D}_{n}\right)\right)\right\}
\end{aligned}
$$

We write $w=w_{N}+w_{T}$ with $w_{N}$ and $w_{T}$ as in (4.5). For simplicity we also define the following matrices:

$$
\begin{aligned}
M_{n} & =\Delta_{W_{n}}\left(w_{T}, \widetilde{\mathbf{D}}_{n}^{0}\right) & M_{n}^{\vartheta} & =\Delta_{W_{n}}\left(\vartheta^{T} w_{T}, \widetilde{\mathbf{D}}_{n}^{0}\right) \\
F_{n} & =2 \operatorname{diag}\left(a_{i}\right)_{i=1}^{n} & F_{n}^{\vartheta} & =2 \operatorname{diag}\left(a_{1}, \ldots, a_{n-1}, 0\right) .
\end{aligned}
$$

We are assuming (remember Remark 4.4) the $a_{n}=\min _{j} a_{j}$. It follows from (3.16), (4.9), (4.10), (4.11) that

$$
\begin{array}{llrl}
\Delta_{W_{n}}\left(w, \widetilde{\mathbf{D}}_{n}^{0}\right) & =M_{n} & \Delta_{W_{n}}\left(\vartheta w, \widetilde{\mathbf{D}}_{n}^{0}\right) & =M_{n}^{\vartheta} \\
\Delta_{W_{n}}\left(w, \mathbf{D}_{n}\right) & =M_{n}+F_{n} & \Delta_{W_{n}}\left(\vartheta w, \mathbf{D}_{n}\right) & =M_{n}^{\vartheta}+F_{n}^{\vartheta} .
\end{array}
$$

By (4.23) and (4.26) we can write

$$
M_{n}^{\vartheta}+F_{n}^{\vartheta}=B_{n-1} \oplus[0]_{1 \times 1}
$$

with $B_{n-1}=\Delta_{W_{n-1}}\left(\vartheta w, \mathbf{D}_{n-1}\right)$. But then we have

$$
M_{n}^{\vartheta}+F_{n}=B_{n-1} \oplus\left[2 a_{n}\right]_{1 \times 1} .
$$


Therefore $^{4}$

$$
\operatorname{spec}\left(M_{n}^{\vartheta}+F_{n}\right)=\operatorname{spec}\left(B_{n-1}\right) \cup\left\{2 a_{n}\right\} .
$$

Since $\vartheta^{T} w_{T}$ is symmetric and positive, by Proposition 4.1(5) the matrix $M_{n}^{\vartheta}$ is positive semidefinite, which implies (see, for instance, [22, Corollary 4.3.3])

$$
\lambda_{k}\left(M^{\vartheta}+F_{n}\right) \geq \lambda_{k}\left(F_{n}\right) \quad k=1, \ldots, n .
$$

Thus we get

$$
2 a_{n}=\lambda_{1}\left(F_{n}\right) \leq \lambda_{1}\left(M_{n}^{\vartheta}+F_{n}\right) .
$$

But (4.28) says that $2 a_{n}$ is actually an eigenvalue of $M_{n}^{\vartheta}+F_{n}$, so it must be the lowest one

$$
2 a_{n}=\lambda_{1}\left(M_{n}^{\vartheta}+F_{n}\right) .
$$

Therefore, by (4.27), we get

$$
\lambda_{2}\left(M_{n}^{\vartheta}+F_{n}\right)=\lambda_{1}\left(B_{n-1}\right)=\lambda_{2}\left(M_{n}^{\vartheta}+F_{n}^{\vartheta}\right) .
$$

Using the explicit expression (3.10) for the matrix elements of the representation $\widetilde{\mathbf{D}}_{n}^{0}$, it is straightforward to check that the matrix elements of $M_{n}-M_{n}^{\vartheta}$ are given by

$$
\left[L_{n}\right]_{i j}:=\left[M_{n}-M_{n}^{\vartheta}\right]_{i j}=\frac{d_{i} d_{j}}{d_{n}},
$$

where $d_{i}=-b_{i n}$ for $i=1, \ldots, n-1$ and $d_{n}=\sum_{i=1}^{n-1} b_{i n}$. Following [10] we observe that $L_{n}$ is a rank-1 matrix, so by standard linear algebra results as [22, Thm. 4.3.4], one obtains, in particular, that

$$
\begin{aligned}
\lambda_{2}\left(M_{n}\right) & =\lambda_{2}\left(M_{n}^{\vartheta}+L_{n}\right) \leq \lambda_{3}\left(M_{n}^{\vartheta}\right) \\
\lambda_{1}\left(M_{n}+F_{n}\right) & =\lambda_{1}\left(M_{n}^{\vartheta}+F_{n}+L_{n}\right) \leq \lambda_{2}\left(M_{n}^{\vartheta}+F_{n}\right) .
\end{aligned}
$$

Thus, using (4.31), we get

$$
\lambda_{1}\left(M_{n}+F_{n}\right) \leq \lambda_{2}\left(M_{n}^{\vartheta}+F_{n}^{\vartheta}\right) .
$$

\footnotetext{
${ }^{4}$ The spectrum is always considered as a multiset, so if, for instance, $\operatorname{spec}(A)=$ $\{0,0,1,2\}$, then $(\operatorname{spec}(A) \backslash\{0\}) \cup\{1\}=\{0,1,1,2\}$.
} 
From (4.21), (4.24), (4.25), (4.26) we obtain

$$
\begin{aligned}
\psi_{W_{n}}\left(w, \mathbf{P}_{n}\right) & =\min \left\{\lambda_{2}\left(M_{n}\right), \lambda_{1}\left(M_{n}+F_{n}\right)\right\} \\
\psi_{W_{n}}\left(\vartheta w, \mathbf{P}_{n-1}\right) & =\min \left\{\lambda_{3}\left(M_{n}^{\vartheta}\right), \lambda_{2}\left(M_{n}^{\vartheta}+F_{n}^{\vartheta}\right)\right\}
\end{aligned}
$$

hence (4.33) and (4.35) imply Lemma 4.7.

\section{A few concluding remarks and one open problem}

Theorem 1.2, together with Proposition 3.1, states that if $w \in \mathcal{A}_{n}$, then the representation "responsible" for the spectral gap is either $\mathbf{D}_{n} \cong[(n-1),(1)]$ or $\widetilde{\mathbf{D}}_{n}^{0}$ that is $[(n-1,1), \emptyset]$, since the trivial summand in (3.13) plays no role. In our proof we are led to consider these two representations because they are the representations which, when restricted to $W_{n-1}$ contain the trivial one.

We show that this is not an artifact of our strategy: we actually need to include both of them, that is the statement of Theorem 1.2 cannot be strengthened by replacing $\mathbf{P}_{n}$ with either $\mathbf{D}_{n}$ or $\widetilde{\mathbf{D}}_{n}^{0}$. Let

$$
w_{N}=\sum_{i=1}^{n} a_{i} s_{\{i\}} \quad w_{T}=\sum_{(i j) \in T_{n}} b_{i j}(i j) \quad a_{i} \geq 0, b_{i j} \geq 0 .
$$

Let $\underline{a}:=\min _{i} a_{i}$ and assume $\underline{a}>0$. Assume also that:

(i) there are enough strictly positive $b_{i j}$ so that $\operatorname{supp}(w)$ generates $S_{n}$.

This condition is equivalent to requiring that the graph on $\{1, \ldots, n\}$ with edge set $\mathcal{E}=\left\{\{i, j\}: b_{i j}>0\right\}$ is connected.

For $\varepsilon>0$ define

$$
w_{\varepsilon}=w_{N}+\varepsilon w_{T} .
$$

Thanks to (4.9), (4.10), (4.11) we can write

$$
\begin{gathered}
F_{n}:=\Delta_{W_{n}}\left(w_{N}, \mathbf{D}_{n}\right)=2 \operatorname{diag}\left(a_{i}\right)_{i=1}^{n} \\
M_{n}:=\Delta_{W_{n}}\left(w_{T}, \mathbf{D}_{n}\right)=\Delta_{W_{n}}\left(w_{T}, \widetilde{\mathbf{D}}_{n}^{0}\right) .
\end{gathered}
$$

Hence

$$
\begin{aligned}
& \Delta_{W_{n}}\left(w_{\varepsilon}, \mathbf{D}_{n}\right)=F_{n}+\varepsilon M_{n} \\
& \Delta_{W_{n}}\left(w_{\varepsilon}, \widetilde{\mathbf{D}}_{n}^{0}\right)=\varepsilon M_{n} .
\end{aligned}
$$


The lowest eigenvalue of $M_{n}$ is trivially $\lambda_{1}\left(M_{n}\right)=0$ with eigenvector equal to any constant vector. It is easy to show that hypothesis (i) above implies that 0 is a simple eigenvalue, that is $\lambda_{2}\left(M_{n}\right)>0$ (see, for instance, Proposition 2.1 of [1]). By perturbation theory we obtain, using (2.4),

$$
\begin{aligned}
& \psi_{W_{n}}\left(w_{\varepsilon}, \mathbf{D}_{n}\right)=\lambda_{1}\left(F_{n}+\varepsilon M_{n}\right)=2 \underline{a}+\mathcal{O}(\varepsilon) \\
& \psi_{W_{n}}\left(w_{\varepsilon}, \widetilde{\mathbf{D}}_{n}^{0}\right)=\varepsilon \lambda_{2}\left(M_{n}\right)=\mathcal{O}(\varepsilon),
\end{aligned}
$$

where $\mathcal{O}(\varepsilon)$ is a generic quantity which goes to 0 as $\varepsilon \rightarrow 0^{+}$. Hence, for small $\varepsilon$ the spectral gap of $w_{\varepsilon}$ is determined by $\widetilde{\mathbf{D}}_{n}^{0}$.

Consider now the opposite situation with

$$
w_{\varepsilon}=\varepsilon w_{N}+w_{T} .
$$

We obtain

$$
\begin{aligned}
& \psi_{W_{n}}\left(w_{\varepsilon}, \mathbf{D}_{n}\right)=\lambda_{1}\left(M_{n}\right)+\mathcal{O}(\varepsilon)=\mathcal{O}(\varepsilon) \\
& \psi_{W_{n}}\left(w_{\varepsilon}, \widetilde{\mathbf{D}}_{n}^{0}\right)=\lambda_{2}\left(M_{n}\right)>0,
\end{aligned}
$$

hence, in this case, for $\varepsilon$ small enough, the spectral gap of $w_{\varepsilon}$ is determined by $\mathbf{D}_{n}$.

Lastly we want to discuss the possibility of proving our main theorem for more general elements $w$ of the group algebra than those considered in (1.7). For $A \subset\{1, \ldots, n\}$, let $s_{A}$ be the element of $W_{n}$ which in the defining representation is given by the diagonal matrix

$$
\mathbf{D}_{n}\left(s_{A}\right)=\operatorname{diag}\left(x_{i}\right)_{i=1}^{n} \quad \text { where } \quad x_{i}= \begin{cases}-1 & \text { if } i \in A \\ +1 & \text { if } i \notin A .\end{cases}
$$

Let then $Y_{n}^{+}\left(Y_{n}^{-}\right)$be the set of all subsets of $\{1, \ldots, n\}$ of even (odd) cardinality, and let

$$
w_{N}^{ \pm}=\sum_{A \in Y_{n}^{ \pm}} a_{A} s_{A} \quad w_{T}=\sum_{(i j) \in T_{n}} b_{i j}(i j) \quad a_{A} \geq 0, b_{i j} \geq 0 .
$$

Question 5.1. Does the equality $\psi_{W_{n}}(w)=\psi_{W_{n}}\left(w, \mathbf{P}_{n}\right)$ also hold for elements $w$ of the form $w=w_{N}^{+}+w_{N}^{-}+w_{T}$ ?

We show that the answer is (in general) negative.

Let

$$
\hat{a}_{i}^{ \pm}=\sum_{A \in Y_{n}^{ \pm}: A \ni i} a_{A}
$$

and assume that: 
(i) there are enough strictly positive $b_{i j}$ so that $\operatorname{supp}(w)$ generates $S_{n}$;

(ii) $w_{N}^{-} \neq 0$;

(iii) $\underline{a}^{+}:=\min _{i} \hat{a}_{i}^{+}>0$.

The first two conditions are necessary, since otherwise the support of $w$ does not generate $W_{n}$, the spectral is trivially zero, and the problem becomes uninteresting.

For $\varepsilon>0$, let

$$
w_{\varepsilon}=w_{N}^{+}+\varepsilon w_{N}^{-}+w_{T} .
$$

Thanks to (4.14) and (4.15) we have

$$
\mathbf{T}^{\beta, \alpha}\left(w_{N}^{+}\right)=\mathbf{T}^{\alpha, \beta}\left(w_{N}^{+}\right) \quad \mathbf{T}^{\beta, \alpha}\left(w_{T}\right)=\mathbf{T}^{\alpha, \beta}\left(w_{T}\right)
$$

which, since $[(n), \emptyset]$ is the trivial representation $\mathbf{I}_{n}$, implies in particular that

$$
\Delta_{W_{n}}\left(w_{N}^{+},[\emptyset,(n)]\right)=\Delta_{W_{n}}\left(w_{T},[\emptyset,(n)]\right)=0 .
$$

As for the "odd term" $w_{N}^{-}$, using (4.15) we obtain

$$
\mathbf{T}^{(\emptyset, \alpha)}\left(s_{A}\right)=(-1) \mathbf{T}^{\alpha}\left(1_{\mathfrak{S}_{n}}\right)=-I_{d},
$$

where $d$ is the degree of the representation $[\alpha]$, thus

$$
\Delta_{W_{n}}\left(w_{N}^{-},[\emptyset, \alpha]\right)=2\left(\sum_{A \in Y_{n}^{-}} a_{A}\right) I_{d} .
$$

From (5.3) and (5.4) it follows that

$$
\psi_{W_{n}}\left(w_{\varepsilon},[\emptyset,(n)]\right)=\varepsilon \lambda_{1}\left(\Delta_{W_{n}}\left(w_{N}^{-},[\emptyset,(n)]\right)\right)=2 \varepsilon \sum_{A \in Y_{n}^{-}} a_{A}=\mathcal{O}(\varepsilon) .
$$

On the other hand we claim that $\psi_{W_{n}}\left(w_{\varepsilon}, \mathbf{P}_{n}\right)$ can be bounded from below by a strictly positive (independent of $\varepsilon$ ) quantity. It is easy to see that equalities (4.9) and (4.10) become

$$
\begin{aligned}
& \Delta_{W_{n}}\left(w_{N}^{ \pm}, \widetilde{\mathbf{D}}_{n}^{0}\right)=0 \\
& \Delta_{W_{n}}\left(w_{N}^{ \pm}, \mathbf{D}_{n}\right)=2 \operatorname{diag}\left(\hat{a}_{i}^{ \pm}\right)_{i=1}^{n} .
\end{aligned}
$$

Let (remember (4.11))

$$
\begin{aligned}
& F_{n}^{ \pm}:=\Delta_{W_{n}}\left(w_{N}^{ \pm}, \mathbf{D}_{n}\right)=2 \operatorname{diag}\left(\hat{a}_{i}^{ \pm}\right)_{i=1}^{n} \\
& M_{n}:=\Delta_{W_{n}}\left(w_{T}, \mathbf{D}_{n}\right)=\Delta_{W_{n}}\left(w_{T}, \widetilde{\mathbf{D}}_{n}^{0}\right) .
\end{aligned}
$$


In this way we have obtained

$$
\begin{aligned}
& \Delta_{W_{n}}\left(w_{\varepsilon}, \mathbf{D}_{n}\right)=M_{n}+F_{n}^{+}+\varepsilon F_{n}^{-} \geq F_{n}^{+} \\
& \Delta_{W_{n}}\left(w_{\varepsilon}, \widetilde{\mathbf{D}}_{n}^{0}\right)=M_{n},
\end{aligned}
$$

where the inequality is intended in the sense of quadratic forms. Thanks to assumption (i) above, we know that $\lambda_{2}\left(M_{n}\right)$ is strictly positive, therefore

$$
\begin{aligned}
\psi_{W_{n}}\left(w_{\varepsilon}, \mathbf{P}_{n}\right) & =\min \left\{\psi_{W_{n}}\left(w_{\varepsilon}, \mathbf{D}_{n}\right), \psi_{W_{n}}\left(w_{\varepsilon}, \widetilde{\mathbf{D}}_{n}^{0}\right)\right\} \\
& \geq \min \left\{\lambda_{1}\left(F_{n}^{+}\right), \lambda_{2}\left(M_{n}\right)\right\}=\min \left\{2 \underline{a}^{+}, \lambda_{2}\left(M_{n}\right)\right\}>0 .
\end{aligned}
$$

Thus, for $\varepsilon$ small enough, we have $\psi_{W_{n}}\left(w_{\varepsilon},[\emptyset,(n)]\right)<\psi_{W_{n}}\left(w_{\varepsilon}, \mathbf{P}_{n}\right)$, which implies a negative answer to Question 5.1.

We observe that a crucial element for this "counterexample" is assumption (iii) above. This leaves room for a conjecture.

Conjecture 5.2. If $w=w_{N}^{-}+w_{T}$, then $\psi_{W_{n}}(w)=\psi_{W_{n}}\left(w, \mathbf{P}_{n}\right)$.

The most obvious approach for proving this result would be to generalize the map $\vartheta^{N}$ of (4.17) as

$$
\vartheta^{N}\left(w_{N}^{-}\right)=\sum_{A \in Y_{n}^{-}: A \not \supset \ell} a_{A} s_{A},
$$

where $\ell$ is the largest index $j$ such that $\hat{a}_{j}^{-}=\min _{i} \hat{a}_{i}^{-}$. Unfortunately this does not work because, with this choice, Lemma 4.7 is false. A counterexample can be found already for $n=3$ : if

$$
w=\sum_{i=1}^{3} s_{\{i\}}+s_{\{1,2,3\}}+(12)+(23)+(13)
$$

then

$$
\vartheta(w)=s_{\{1\}}+s_{\{2\}}+\frac{3}{2}
$$

which produces $\psi_{W_{3}}\left(w, \mathbf{P}_{3}\right)=3>2=\psi_{W_{2}}\left(\vartheta(w), \mathbf{P}_{2}\right)$. We emphasize that this is a counterexample to Lemma 4.7, not to Conjecture 5.2, since we have in fact $\psi_{W_{3}}(w)=3$. Hence one should devise a different map $\vartheta: \mathcal{A}_{n} \rightarrow \mathcal{A}_{n-1}$, keeping in mind that there is a delicate balance between the two properties (a) and (b) of Section 4 which must be satisfied by $\vartheta$.

Acknowledgements. In the first version of this paper we erroneously claimed to have proven Conjecture 5.2 due to a mistake in the proof of (a more general version of) Lemma 4.7 where (5.7) was used. We thank one of the referees for finding the mistake in the proof, which prompted us to find the above counterexample. 


\section{References}

[1] F. Cesi, A few remarks on the octopus inequality and Aldous' spectral gap conjecture, Communications in Algebra 44 (1) (2016) 279-302.

[2] P. Diaconis, M. Shahshahani, Generating a random permutation with random transpositions, Z. Wahrsch. Verw. Gebiete 57 (2) (1981) 159179.

[3] L. Flatto, A. M. Odlyzko, D. B. Wales, Random shuffles and group representations, Ann. Probab. 13 (1) (1985) 154-178.

[4] R. Bacher, Valeur propre minimale du laplacien de Coxeter pour le groupe symétrique, J. Algebra 167 (2) (1994) 460-472.

[5] S. Handjani, D. Jungreis, Rate of convergence for shuffling cards by transpositions, J. Theoret. Probab. 9 (4) (1996) 983-993.

[6] T. Koma, B. Nachtergaele, The spectral gap of the ferromagnetic XXZ chain, Lett. Math. Phys. 40 (1) (1997) 1-16.

[7] B. Morris, Spectral gap for the interchange process in a box, Electron. Commun. Probab. 13 (2008) 311-318.

[8] S. Starr, M. P. Conomos, Asymptotics of the spectral gap for the interchange process on large hypercubes, Journal of Statistical Mechanics: Theory and Experiment 2011 (10) (2011) P10018.

[9] F. Cesi, On the eigenvalues of Cayley graphs on the symmetric group generated by a complete multipartite set of transpositions, J. Algebraic Combin. 32 (2) (2010) 155-185.

[10] A. B. Dieker, Interlacings for random walks on weighted graphs and the interchange process, SIAM J. Discrete Math. 24 (1) (2010) 191-206.

[11] P. Caputo, T. M. Liggett, T. Richthammer, Proof of Aldous' spectral gap conjecture, J. Amer. Math. Soc. 23 (3) (2010) 831-851.

[12] D. Aldous, www.stat.berkeley.edu/\%7Ealdous/Research/OP/sgap.html.

[13] F. Cesi, Cayley graphs on the symmetric group generated by initial reversals have unit spectral gap, Electron. J. Combin. 16 (1) (2009) Note 29, 7 . 
[14] X. Huang, Q. Huang, The adjacency spectral gap of some cayley graphs on alternating groups, arXiv:1711.08944 (2017).

[15] F. Chung, J. Tobin, The spectral gap of graphs arising from substring reversals, The Electronic Journal of Combinatorics 24 (3) (2017) 3-4.

[16] O. Parzanchevski, D. Puder, Aldous' spectral gap conjecture for normal sets, arXiv:1804.02776 (2018).

[17] M. Kassabov, Subspace arrangements and property T, Groups, Geometry, and Dynamics 5 (2) (2011) 445-477.

[18] M. Geck, G. Pfeiffer, Characters of finite Coxeter groups and IwahoriHecke algebras, Vol. 21 of London Mathematical Society Monographs. New Series, The Clarendon Press Oxford University Press, New York, 2000 .

[19] G. James, A. Kerber, The representation theory of the symmetric group, Vol. 16 of Encyclopedia of Mathematics and its Applications, Addison-Wesley Publishing Co., Reading, Mass., 1981.

[20] L. Geissinger, D. Kinch, Representations of the hyperoctahedral groups, Journal of algebra 53 (1) (1978) 1-20.

[21] J.-P. Serre, Linear representations of finite groups, Vol. 42, Springer, 1977.

[22] R. A. Horn, C. R. Johnson, Matrix analysis, Cambridge University Press, Cambridge, 1990, corrected reprint of the 1985 original. 